\title{
Comments on the Nobel Prize Awarded to Roger Penrose
}

\section{Y. Lo}

Applied and Pure Research Institute, 15 Walnut Hill Rd., Amherst, NH 03031

\begin{abstract}
The Nobel Prize Committee for Physics awarded the 2020 Nobel Prize to Roger Penrose, Reinhard Genzel, and Andrea Ghez for their discoveries about the black holes. Genzel and Ghez discovered that an invisible and extremely heavy object governs the orbits of stars at the centre of our galaxy. However, Penrose's work is outdated because gravity is no longer always attractive since repulsive gravity was discovered in 1997 and subsequently confirmed by experiments. Thus, repulsive gravitation does exist, and Einstein, Newton, Galileo and Maxwell are incorrect. Relativists, including Einstein, are often carelessly overlooked simple, but subtle theoretical errors such as $\mathrm{E}=\mathrm{mc}^{2}$. Since gravity was not always attractive, it is necessary to rejustify whether black hole really exist, in spite of the existence of repulsive gravitation. Moreover, repulsive gravitation force would necessary extend general relativity to a five-dimensional theory, which is beyond the understanding of Penrose and the Nobel Committee. In conclusion, the award of a Nobel Prize to Penrose is really groundless since there is no clear evidence for the existence of the black holes.
\end{abstract}

Keywords: current-mass interaction; charge-mass interaction; repulsive gravitation; $\mathrm{E}=$ $\mathrm{mc}^{2}$. 
PACS: 04.20.Cv; 04.50.-h; 04.50.Kd; 04.80.Cc

\section{Introduction}

The Nobel Prize Committee for Physics awarded the 2020 Nobel Prize to Roger Penrose, Reinhard Genzel, and Andrea Ghez for their discoveries about the black holes. The prize divided into two, with one half going to Penrose, and the other half jointly to Genzel and Ghez [1].

Genzel and Ghez [2] discovered that an invisible and extremely heavy object governs the orbits of stars at the centre of our galaxy, but whether it is a supper black hole is not clear. This is a true scientific discovery and certainly deserve a prize. However, the claim that "UKborn Penrose showed that Albert Einstein's general theory of relativity leads to the formation of black holes." is questionable as an achievement in physics. The problem is that Penrose's work, as usual, is not verifiable [3]. Physics prize should go to verifiable results because as Galileo taught us if a conclusion in physics can pass the test of real experiments, then we can be sure that it at least partially valid in physics. ${ }^{1)}$ However, since repulsive gravitation has been confirmed [3], Penrose must show that black hole still exists in spite of this.

\section{The Conditional Validity of $E=m c^{2}$}

A problem of general relativity is that relativists are often careless, starting from Einstein. For instance, it is generally considered that the Einstein equation and the formula $\mathrm{E}=\mathrm{mc}^{2}$ are two great achievements. However, they failed to see that these two equations are not even consistent. This would explain why the 2020 Nobel Committee made errors and failed to see them.

The formula $E=m c^{2}$ in special relativity only means that mass can be converted into energy. Einstein wants to have new content, $m=E / c^{2}$, i.e., any energy $\mathrm{E}$ can be equivalent to mass. However, Einstein failed, although he made a great effort to prove this between 1905-1909 [4]. In fact, the invalidity of $\mathrm{E}=\mathrm{mc}^{2}$ can be proved.

For the electromagnetic energy $E, E=m c^{2}$ is inconsistent with the Einstein equation $[5,6]$,

$$
G_{\mu \nu} \equiv R_{\mu \nu}-(1 / 2) g_{\mu \nu} R=-K T_{\mu \nu},
$$


where $G_{\mu \nu}$ is the Einstein tensor, $R_{\mu \nu}$ is the Ricci tensor, $R=R_{\mu v} g^{\mu \nu}$ is the Ricci curvature, $T_{\mu \nu}$ is the sum of energy-stress tensor, and $K$ is the coupling constant. Then, we have

$$
R=K T_{\mu \nu} g^{\mu \nu}
$$

Note that eq. (2) is completely general.

For the case of electromagnetic energy $E$, the trace of the electromagnetic stress tensor $T(E)_{\mu \nu}$ is zero, i.e., $g^{\mu v} T(E)_{\mu \nu}=0$. Thus, it cannot change the Ricci curvature R. However, the mass $m$ is able to do so since the trace for the massive energy-stress tensor is non-zero. Thus, electromagnetic energy and mass are not equivalent. Since eq. (2) was first derived by Einstein [6], the failure of seeing this inconsistency with $E=m c^{2}$ is Einstein's careless oversight. Thus, Einstein's theory alone would show that $E=m c^{2}$ is not always valid, independent of the existence of repulsive gravitation.

Since Hawking [7] believed the invalid $\mathrm{E}=\mathrm{mc}^{2}$, he has no verified results. Although the photonic energy is equivalence to mass, it is not pure electromagnetic energy. Thus, in 1905 Einstein regarded the photonic energy is a quanta of electromagnetic energy [8] is only partially true. The photons as massless particles [9], is inconsistent with electromagnetic energy [10].

The photonic energy is a combination of the energy of the electromagnetic wave energy and the related gravitational wave energy $[11,12]$. Thus, the energy of photons is not pure electromagnetic energy as shown by the following equations:

$$
G_{a b} \equiv R_{a b}-(1 / 2) g_{a b} R=-K\left[T(w)_{a b}-T(p)_{a b}\right]
$$

and

$$
T_{a b}=-T(g)_{a b}=T(w)_{a b}-T(p)_{a b},
$$

where $T(w)_{a b}$ and $T(p)_{a b}$ are the energy-stress tensors for the electromagnetic wave and the related photons, which are massless particles.

Thus, the photonic energy must also include the energy of its gravitational-wave component. The energy, related to the photons, is clearly beyond special relativity. Moreover, the implicit assumption of a unique sign for all coupling constants in space-time singularity theorems is invalid. Thus, the claim of Hawking and Penrose that general relativity is not suitable for microscopic phenomena is simply incorrect. 
Although Dmitriev, Nlkushchenko, \& Snegov [13] and Fan, Feng \& Liu [14] obtain the correct weight reduction for a piece of metal as temperature increases, they had mistaken this as a reduction of mass. Some went to the extreme that electromagnetic energy would lead to a reduction of weight. This is also not true because the current-mass interaction would generate attractive gravitational force [3]. Such a force has been discovered by Martin Tajmar and Clovis de Matos [15] from the European Space Agency.

Moreover, Einstein justified a curve space by showing the ratio $U / D>\pi$, (where $U$ and $D$ are, respectively, the circumference and the diameter of a circle in the rotating frame) with applications of special relativity [16]. Since it has nothing to do with gravitation, it is difficult to reconcile this and that Einstein proposed special relativity. Thus, there must be mistakes in Einstein's application [16].

In view of the facts that so many simple but subtle errors are overlooked, one should not be surprised that Penrose, essentially a mathematician, was awarded the 2020 Nobel Prize.

\section{The Question of Black Holes and Repulsive Gravitation}

The existence of black holes is a mathematical result obtained by simulation under the assumption that gravity was always attractive [17]. However, it is well-known that this assumption is no longer valid because repulsive gravitation has been discovered in 1997 [18]. Subsequently repulsive gravitation is confirmed by several experiments $[19,20]$. Because experiment shows that heated-up metals have a reduced weight [21], instead of increased weight [22] as Einstein predicted, Einstein's theory clearly has errors. Thus, results from general relativity should be reviewed.

Moreover, the papers $[23,24]$ cited by the Nobel Committee are published in 1963 and 1965, Since the existence of repulsive gravitation was discovered in 1997, Penrose's knowledge on gravitation was clear out-dated. Furthermore the existence of the repulsive gravitational force implies that general relativity must be extended to a five-dimensional theory [3]. This is clearly beyond Penrose, whose ability is limited to four-dimensional theory. Hence, Penrose would be unable to rejustify the validity of the black hole notion. Thus, one cannot claim general relativity leads to the formation of black holes.

Moreover, for a particle with charge $\mathrm{q}$, the repulsive gravitational force to a mass $\mathrm{m}$ at a distance $\mathrm{r}$ is $\mathrm{mq}^{2} / \mathrm{r}^{3}$ that would become stronger than a gravitational force at very small 
distance. This would prevent the formation of a singularity. This would explain why a black hole has never been observed because it does not exist. For more details, the question of black hole must be studied together with repulsive gravitation.

In conclusion, the award of a Prize to Penrose is really groundless. Since the knowledge of the Nobel Committee on gravitation is out-dated, the Committee is really not yet ready to give a prize in this area. I do hope the Committee to show clearly that the existence of black hole is beyond our understanding in physics. Furthermore, the Committee should up-dated her knowledge on gravitation.

Moreover, since the existence of a black hole has not been proved, the Nobel Committee should encourage to obtain a clear answer for this problem in the future. We should learn from the lessons that relativists often make mistakes without knowing them and relativity must be reviewed thoroughly. They should also encourage research in repulsive gravitation for the progress of physics as Nobel expected

\section{Acknowledgments}

The paper is dedicated to Dr. S. Hockfield, the 16th President of MIT, for her support and encouragement on investigation of repulsive gravitation. I would like to thank Prof. S. Weinberg, who taught me general relativity at MIT, and Prof. I. Halperin, who taught me mathematics at Queen's University, Canada. The author is grateful to G. P. Wood and S, Holcombe for their valuable suggestions and comments. This work is supported, in part, by the Chan Foundation, and the Szecheon Co., Hong Kong, China.

\section{Appendix A: The Principle of Causality in Physics}

Physics is essentially a science for causality. There are two aspects in causality: its relevance and its time ordering. In time ordering, a cause event must happen before its effects. This is further restricted by relativistic causality that no cause event can propagate faster than the light speed in the vacuum. The time-tested assumption that phenomena can be explained in terms of identifiable causes will be called the principle of causality. This is the basis of relevance for all scientific investigations.

Causality means causes will lead to consequences. It should be emphasized that the principle assumed: 
1) From the consequences that causes must exist even we do not know what they are.

2) The partial consequences of the cause are identified even its full consequences remain to be known.

Then, we can use such partial consequences as requirements to decide whether a solution or even an equation is valid in physics. This might often provide crucial steps to solve a problem correctly. For example, this is how the equation (3) for the electromagnetic wave as a source was modified.

Thus, this principle implies that any parameter in a solution for physics must be related to some physical causes. Moreover, the principle of causality implies that a weak source would produce a weak gravity. Here this principle will be elucidated first in connection with symmetries of a field, the boundedness of a field solution. Although this principle alone cannot derive a field equation or its solution, it can help determine whether they are valid. This has made a difference in the investigation of gravitation $[3,11,12,25,26]$.

In practice, when the considered field is absent, physical properties are ascribed to the spacetime as in a "normal" state. For example, the electromagnetic field is zero in a normal state. Then, any deviation from the normal state must have physically identifiable causes. Thus, the principle of causality implies that the symmetry must be preserved if no cause breaks it. The implication of causality to symmetry has been used in deriving the inverse square law from Gauss's law. The normal state of a space-time metric is the flat metric in special relativity. Thus, if a metric does not possess a symmetry, then there must be a physical cause(s) which has broken such a symmetry. For a spherically symmetric mass, causality requires that the metric is spherically symmetric and asymptotically flat. Also, a weak cause can lead to only weak gravity. Thus, Einstein's weak gravity is a consequence of causality.

However, the physical cause(s) should not be confused with the mathematical source term in the field equation. In general relativity, the cause of gravity is the physical matter itself, but not its energy tensors in the source term of Einstein's field equation. The energy-stress tensors (for example, the perfect fluid model) may explicitly depend on the metric. Since nothing should be a cause of itself, such a source tensor does not represent the cause of a metric. For the accompanying gravitational wave of an electromagnetic wave, the physical cause is the electromagnetic wave. Thus, one should not infer the symmetries of the metric based on the source term instead of its causes. 
Moreover, inferences based on the source term can be misleading because it may have higher symmetries than those of the cause and the metric. For instance, a transverse electromagnetic plane-wave is not rotationally invariant with respect to the $z$-direction of propagation. But the related electromagnetic energy-stress tensor component $T(E)_{t t}$ for a circularly polarized wave is. Such an assumption violates causality and results in theoretical difficulties.

A reason that the Einstein equation did not have a bounded dynamic solution is its violation of causality. In the Einstein equation the left side is the Einstein tensor $G_{\mu \nu}$ and the right side are the energy-momentum tensors. For the dynamic case, the energy-momentum tensor of the gravitational waves should have been included. Thus, for the dynamic case, the Einstein equation violates the principle of causality and thus has no bounded dynamic solution. The modified Einstein equation (8) can have a dynamic solution because the missing energymomentum tensor has been added back. It is surprising that physicists did not find this principle of causality for general relativity earlier.

Classical electrodynamics implies that the flat metric is an accurate approximation, caused by the presence of weak electromagnetic waves. This physical requirement is supported by the principle of causality, which implies such a metric to be a bounded periodic function. However, this required

boundedness is not satisfied with many solutions in the literature [27-39]. If these authors understood the

principle of causality, they would not have produced them.

Many theorists and journals do not understand this principle adequately. For instance, the Physical Review accepted an unbounded solution as valid in physics. As well, the Royal Society (London) accepted Hawking, even though the space-time singularity theorems violate the principle of causality. A major problem is that the teaching of Galileo on the importance on experimental verification is often forgotten.

\section{Endnotes}

1) The pass of an experiment test, confirms at least some part of the conclusion. For instance, Einstein's photon is confirmed as an energy quanta although it has not been confirmed as entirely constructed by electromagnetic energy. 
2) Both Dmitriev et al. [13] and Fan et al. [14] had mistaken a reduction of weight as a reduction of mass.

\section{References:}

[1] Announcements of the 2020 Nobel Committee (2020).

[2] R. Genzel, F. Eisenhauer, and S. Gillessen, "The Galactic Center massive black hole and nuclear star cluster," Rev. Mod. Phys. 82, 3121(2010).

[3] C. Y. Lo, Incompleteness of general relativity, Einstein's errors, and related experiments, American Physics Society March Meeting, Z23 5, 2015, Journal of Advances in Physics 8(2) (2015), 2135-2147.

[4] Einstein's Miraculous Year, edited by John Stachel, Princeton University Press, Princeton, 1998.

[5] A. Einstein, H. A. Lorentz, H. Minkowski and H. Weyl, The Principle of Relativity, Dover, New York, 1923.

[6] A. Einstein, The Meaning of Relativity, Princeton Univ. Press, 1954.

[7] S. W. Hawking, A Brief History of Time- from big bang to black hole (Random House Publishing Group,1998).

[8] A. Einstein, On a heuristic point of view concerning the production and transformation of light, Annalen der Physik 17 (1905), 132.

[9] A. Einstein, Does the inertia of a body depend upon its energy-content?, Annalen der Physik 18 (1905), 639.

[10] C. Y. Lo and Richard C. Y. Hui, Physics Essays 31(1) (2018).

[11] C. Y. Lo, The gravity of photons and the necessary rectification of Einstein equation, Progress in Physics 1 (2006), 46-51.

[12] C. Y. Lo, Completing Einstein's proof of $E=m c^{2}$, Progress in Physics 4 (2006), 14-18.

[13] A. L. Dmitriev, E. M. Nikushchenko and V. S. Snegov, Influence of the temperature of a body on its weight, Measurement Techniques 46(2) (2003), 115-120.

[14] Fan Liangzao, Feng Jinsong and Liu Wu Qing, Engineer Sciences 8(2) (2010), 9-11.

[15] http://news.softpedia.com/news/The-First-Test-That-Proves-General-Theory-ofRelativity-Wrong-20259.shtml.

[16] C. Y. Lo, Rectifiable Inconsistencies and Related Problems in General Relativity, Physics Essays 23(2) (2010). 
[17] K. S. Thorne, Black Holes and Time Warps, Norton, New York, 1994.

[18] C. Y. Lo, Astrophys. J. 477 (1997), 700-704.

[19] C. Y. Lo, Gravitation, physics, and technology, Physics Essays 25(4) (2012), 553-560.

[20] C. Y. Lo, The Invalid Speculation of $\mathrm{m}=\mathrm{E} / \mathrm{c}^{2}$, the Reissner-Nordstrom Metric, and Einstein's Unification, Phys. Essays, 25 (1), 49-56 (2012).

[21] C. Y. Lo, The temperature dependence of gravitation for the metallic balls - Measured with a torsion balance scale, GJSFR-F 17(4) (2017), 1-16, Ver. 1.0.

[22] Einstein, $E=m c^{2}$, (1946), Ideas and Opinions, p. 337, Crown, New York, 1982.

[23] R. Penrose, "Asymptotic Properties of Fields and Space-Times," Phys. Rev. Lett. 10, 66 (1963).

[24] R. Penrose, "Gravitational Collapse and Space-Time Singularities," Phys. Rev. Lett. 14, 57 (1965).

[25] C. Y. Lo, Einstein's Radiation Formula and Modifications to the Einstein Equation, Astrophysical Journal 455 (1995), 421-428; Editor S. Chandrasekhar suggests the appendix therein.

[26] C. Y. Lo, On incompatibility of gravitational radiation with the 1915 Einstein equation, Physics Essays 13(4) (2000), 527-539.

[27] A. Peres, Phys. Rev. 118 (1960), 1105.

[28] W. B. Bonnor, Commun. Math. Phys. 13 (1969), 163.

[29] R. Bartnik, J. Math. Phys. 38 (1997), 5774.

[30] S. Frittell and E. T. Newman, Phys. Rev. D 55 (1997), 1971.

[31] E. Malec, J. Math. Phys. 38 (1997), 3650.

[32] G. A. Burnett and A. D. Rendall, Class. Quant. Grav. 13 (1996), 111.

[33] H. Friedrich, Class. Quant. Grav. 13 (1996), 1451.

[34] P. Hubner, Phys. Rev. D 53 (1996), 701.

[35] A. D. Rendall, Helv. Phys. A 69 (1996), 490.

[36] P. R. Brady, Phys. Rev. D 51 (1995), 4168.

[37] Hubner, Class. Quant. Grav. 12 (1995), 791.

[38] J. Jezierk, Gen. Relat. and Grav. 27 (1995), $821 .$.

[39] U. Brauer, A. Rendall and O. Reula, Class. Quant. Grav. 11 (1994), 2283 